\title{
PHYSICAL AND ELECTROCHEMICAL CHARACTERIZATION OF CATALYSTS FOR OXYGEN REDUCTION IN FUEL CELLS
}

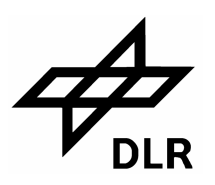

\author{
C.Christenn, M. Schulze, G. Steinhilber, T. Kaz, K. A. Friedrich \\ German Aerospace Center (DLR), Institute of Technical Thermodynamics \\ Pfaffenwaldring 38-40, D-70569 Stuttgart, Germany, Claudia.Christenn@dlr.de
}

\section{Introduction}

In low temperature fuel cells the oxygen reduction reaction (ORR) at the cathode is responsible for a large part of the losses of the conversion from chemical to electrical energy. Especially for the reaction kinetics the catalyst is the crucial factor, while the structure of the electrodes is important for catalyst utilization, efficient mass transport and durability of the cell. In particular, the cathode catalysts currently used are associated with major cell efficiency losses, because of the kinetic limitation of the oxygen reduction reaction.

To solve this problem a better understanding of the key steps in the oxygen reduction process on commonly used cathode catalysts as well as of alternative catalysts is required. For this purpose physical and electrochemical methods are used to characterize the catalysts. Besides the $\mathrm{V}$-i-characteristics and the electrochemical impedance spectroscopy (EIS) as electrochemical methods, the physical methods porosimetry by mercury intrusion and temperature programmed reduction (TPR) are used to characterize the catalysts. Additionally, surface science analytical methods such as x-ray photoelectron spectroscopy (XPS) provide important insights into the structural and chemical properties of the catalyst. In order to perform a complete surface science analysis of the catalysts it is not sufficient to investigate freshly prepared catalysts, but also necessary to study the catalysts after operation in fuel cells or after electrochemical treatment. Comparing the catalysts before and after the electrochemical stressing the alterations of the catalysts can be determined.

As an example the results of combined investigations of carbon supported ruthenium-selenide cathode catalysts operated in a DMFC are presented here.

\section{Carbon supported RuSe- catalysts}

\section{Following catalysts are compared:}

- 40 wt \% RuSe/C (26.0 wt.\% Ru, 13.4 wt. \% Se) (TUM)

- 20 wt\% RuSe/C (18.7 wt.\% Ru, 0.9 wt. \% Se) (HMI)

- $40 \mathrm{wt} \% \mathrm{Pt} / \mathrm{C}$ (Reference catalyst)

\section{Physical characterization}

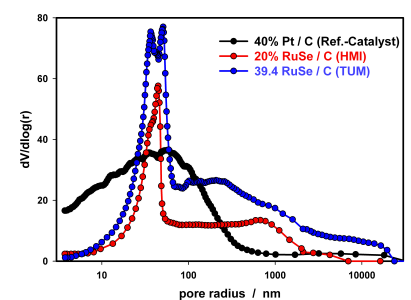

Fig. 1: Pore size distribution of carbon supported and $\mathrm{Pt}$

(by Hg-porosimetry)

RuSe/C catalysts have a pore system at $30-50 \mathrm{~nm}$, that is mainly dominated by the carbon support (Vulcan $\mathrm{XC72).}$

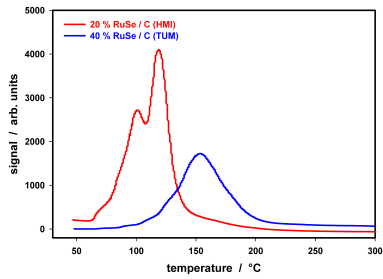

Fig. 2: TPR-spectra of RuSe/C catalysts RuSe/C catalysts
(reduction tempera20 wt $\%$ Ruse/C $20 \mathrm{wt} \%$
$120^{\circ} \mathrm{C} ;$ $40 \mathrm{wt} \%$ RuSe/C: $\left.154^{\circ} \mathrm{C}\right)$
Higher reduction temperature of $40 \mathrm{wt}$ \% RuSe/C catalyst is caused by the higher $\mathrm{Se}$ to $\mathrm{Ru}$ ratio (a more stable oxide).

\section{Electrochemical characterization}

Single cell tests of RuSe/C DMFC cathodes
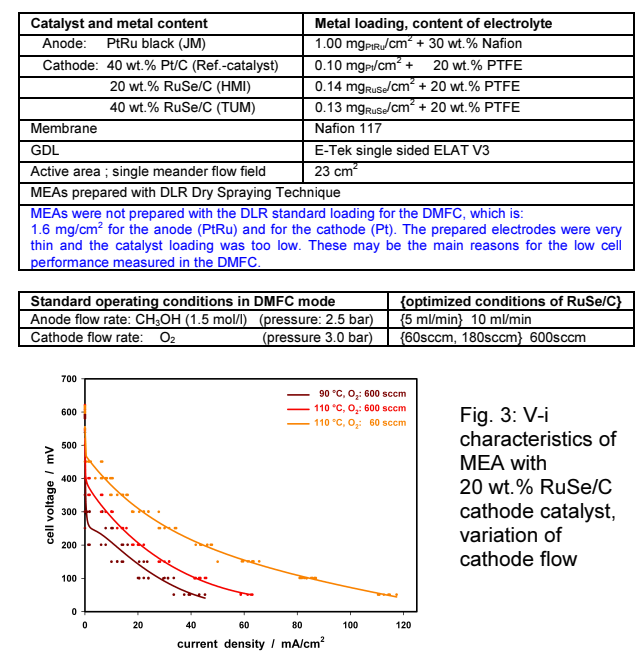

Fig. 3: V-i characteristics of MEA with 20 wt. \% RuSe/C cathode catalyst, variation of cathode flow

Increase of the cell temperature and a lower oxygen flow rate lead to an increase of the cell performance. RuSe/C catalysts allow a higher water content in the cathode. This indicates a different hydrophobic character compared to Pt catalyst or an improved water transport.

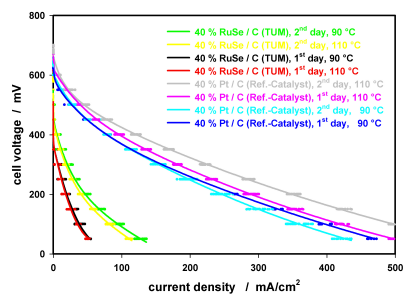

Fig. 4: Change of $V-i$ characteristics from $1^{\text {st }}$ to $2^{\text {nd }}$ day

The general increase of cell performance from $1^{\text {st }}$ to $2^{\text {nd }}$ day for RuSe/C catalyst is caused by an improved water balance. In contrast, the performance of the MEA with the $\mathrm{Pt}$ catalyst is increased at $110^{\circ} \mathrm{C}$, whereas the performance at $90^{\circ} \mathrm{C}$ is decreased. This behavior of the MEAs with the Pt and with the RuSe catalysts indicates that the catalysts have significantly different hydrophobic properties.

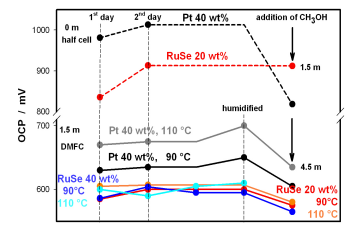

Fig. 5: Open circuit potential (OCP) measured for the Pt and the RuSe cathode in half cell configuration ( $1 \mathrm{~m}:=\mathrm{mol} / / \mathrm{H}_{2} \mathrm{SO}_{4}$ ) and open cell voltage measured in the DMFC

OCP of MEA with RuSe catalyst is lower compared to $\mathrm{Pt}$ catalyst. Voltage drop for RuSe due to increased $\mathrm{CH}_{3} \mathrm{OH}$ concentration indicates an anodic effect. The anode reaction must also be influenced by the different cathode catalysts, maybe by the formation of $\mathrm{H}_{2} \mathrm{O}_{2}$ on the cathode, which can crossover the membrane to the anode, where a mixed potential can be formed.

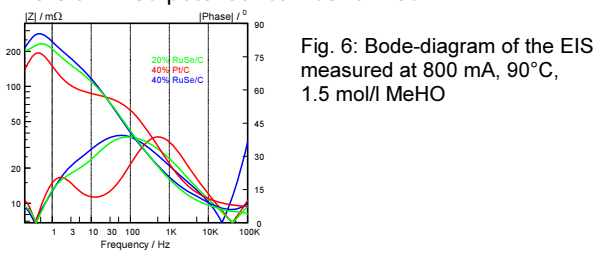

The cell with 40 wt $\% \mathrm{Pt} / \mathrm{C}$ cathode shows the highest catalytic activity. Electrode with $20 \mathrm{wt} \% \mathrm{RuSe} / \mathrm{C}$ has a higher catalytic activity compared to the $40 \mathrm{wt} \% \mathrm{RuSe} / \mathrm{C}$ cathode. Minor differences in the high-frequency range of the diagram are caused by a different conductivity of the electrode material.

\section{Physical characterization - post mortem}

XPS investigation of RuSe/C catalysts before and after the electrochemical stressing
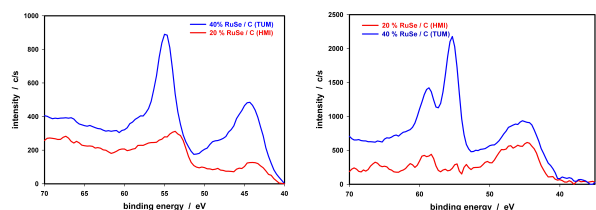

Fig. 7: Se 3d (and Ru 4p) spectra of new (right) and electrochemically stressed (left) electrodes with RuSe/C catalyst

Increase of Se 3d peak of 20 wt\% RuSe/C catalyst after operation indicates cleaning effect. Se is present in two forms: as selenides and in oxidized form. Significant decrease of the selenium oxide peak of $40 \mathrm{wt} . \% \mathrm{RuSe} / \mathrm{C}$ catalyst was measured after stressing.

\section{Conclusions}

The application of the selected methods for physical and electrochemical characterization leads to useful results for further development of new catalyst systems.

- Catalyst composition and MEA structure change due to electrochemical stressing (DMFC and XPS measurements)

- Reaction kinetics of $\mathrm{Pt}$ and RuSe cathodes are different (as deduced from EIS measurements)

Methanol tolerance of RuSe catalysts is confirmed, increased methanol concentrations affect Pt anodes (OCP in DMFC and half cell experiments)

Surface composition of catalysts depends on support and preparation (XPS, Hg-porosimetry)

\section{Acknowledgment}

Thanks to the partners of the network for the supply of the catalysts:

S. Fiechter, P. Bogdanoff, I. Dorbandt, I. Hermann from Hahn-Meitner-Institut (HMI) Berlin,

U. Stimming, A. Racz from Technische Universität München (TUM)

Funding by the "Efficient Oxygen Reduction for the Electrochemical Energy Conversion - $\mathrm{O}_{2}$ RedNet" - network by the German Ministry of Education and Research is gratefully acknowledged. 